\title{
MODELLOS DE REGRESIÓN PARA ESTIMAR EL PESO SECO DE ÓRGANOS Y ÁREA DEL LIMBO DEL DURAZNERO, VARIEDAD JARILLO
}

\section{REGRESSION MODELS FOR THE ESTIMATION OF THE DRY WEIGHTS OF ORGANS AND THE LIMBO AREA OF THE PEACH VARIETY JARILLO}

\author{
Enrique Quevedo García ${ }^{1}$, Giovanni Orlando Cancino Escalante ${ }^{2}$, Anderson Rodrigo Barragán Torres ${ }^{3}$
}

\begin{abstract}
${ }^{1}$ Ing. Agrónomo M.Sc. Profesor titular Departamento de Agronomía, Programa de Ingeniería Agronómica. Universidad de Pamplona. Doctorando en Ciencias Naturales para el Desarrollo énfasis en sistemas de producción agrícola. Universidad Nacional de Costa Rica. Tecnológico de Costa Rica y Universidad Estatal a Distancia, Heredia (Costa Rica), e-mail: enriquegarcia@unipamplona.edu.co; ${ }^{2}$ Biólogo Ph.D., Profesor titular Departamento de Biología, Programa de Biología y Maestría en Biología molecular y biotecnología. Universidad de Pamplona, km 1 vía Bucaramanga-Pamplona, Norte de Santander, e-mail: gcancino@unipamplona.edu.co; ${ }^{3}$ Ingeniero Agrónomo. Universidad de Pamplona, e-mail: anderson. barragan@unipamplona.edu.co
\end{abstract}

Rev. U.D.C.A Act. \& Div. Cient. 20(2): 299-310, Julio-Diciembre, 2017

\section{RESUMEN}

Los métodos no destructivos y confiables para estimar el área del limbo y el peso seco de los órganos de durazno de la variedad Jarillo están limitados para el investigador. Por el contrario, en este estudio, se desarrollaron unos modelos estadísticos para estimar el área y el peso seco del limbo, de frutos y de ramas de la variedad Jarillo, para ser utilizados, como herramientas, para facilitar la recolección de datos. El estudio, se realizó en las montañas de Pamplona, noroeste de Colombia. Se seleccionaron 240 hojas, de 2,5 meses; 240 frutos, de 3,5 y 7 meses y 240 ramas, de 9 meses de edad, para desarrollar los modelos. Los resultados del análisis de regresión indicaron que varios modelos son adecuados para estimar el área del limbo y el peso seco de los órganos de durazno de la variedad Jarillo. Los datos observados y estimados se compararon estableciendo la relación, mediante una regresión lineal simple, para la rama y el limbo. La relación para el fruto fue de tipo cuadrático. El número de yemas, de volumen, de longitud y el diámetro basal/medio de las ramas mixtas, el peso seco del fruto, el muestreo, el diámetro longitudinal del fruto, la altitud, la longitud del limbo, el peso seco del limbo, el producto de la longitud por el ancho del limbo, el ancho del limbo, el peso seco del limbo, están involucrados en la estimación del área del limbo y el peso seco de los órganos de Prunus persica (L.), variedad Jarillo.

Palabras clave: Medidas lineales, dimensiones foliares, predicciones, ramas, modelado, peso seco.

\section{SUMMARY}

Non-destructive, rapid and reliable methods for estimating the area of limb and dry weight of peach organs for the Jarillo variety are limited. Therefore, the aim of the present study was to produce a statistical model to estimate the area and dry weight of limb fruit and branches of this specific variety that can be used as a tool to facilitate data collection. The study was performed in the mountains of Pamplona, Northwest of Colombia. Two Hundred and forty leaves (240) from 2.5 months old limb, 240 fruits of 3.5 and 7 months old fruits and 240 branches of 9 months old branches were selected and used to develop the model. Results of the regression analysis indicated that several models were suitable to estimate the area limb and the dry weight. The observed and estimated data were compared by establishing the relationship by means of a simple linear regression for the branch and limb. The relation for the fruit was quadratic type. The number of buds, volume of branches, length of mixed branches and basal/mean diameter, dry weight of fruit, sampling, longitudinal diameter of the fruit, altitude, length of limb, dry weight of limb, the product of the length by the width of the limb, width of the limb, should be considered in the estimation of the area limb and dry weight of the peach organs of $P$. persica (L.) Jarillo variety.

Key words: Linear measurements, leaf dimensions, predictions, branches, modelling; dry weight. 


\section{INTRODUCCIÓN}

El Prunus persica L. (Batsch) es uno de los frutales comúnmente plantado en zonas del trópico alto, debido a que posee ventajas comparativas con los que se plantan en las zonas templadas (Pinzón et al. 2014). La producción del duraznero en Colombia, se concentra en los departamentos de Boyacá, Cundinamarca, Norte de Santander y Santander, ubicados en la cordillera oriental, en municipios localizados en la franja altitudinal, entre los 1.600 y $3.000 \mathrm{msnm}$ (Carranza et al. 2013). El cultivar Jarillo es de origen venezolano y es sembrado, principalmente, en el departamento de Norte de Santander (Campos, 2013).

Como factor ambiental, la altitud en las localidades ejerce efectos profundos sobre la fisiología de la planta, a través del aumento de la radiación, especialmente ultravioleta y el viento, mientras que la temperatura disminuye $0,6^{\circ} \mathrm{C}$ cada $100 \mathrm{~m}$ de altitud y la precipitación (a partir de 1.300$1.500 \mathrm{msnm}$ ), se reduce (Fischer, 2005). También, Fischer et al. (2012) indicaron que a mayor altitud tenga el piso térmico (a partir de $1.500 \mathrm{msnm}$ ), se tiene menor humedad relativa. Correctas predicciones del limbo, que es la parte de la hoja sin el peciolo y que en plantas individuales permiten indagar en aspectos, como la plasticidad fenotípica, para mejorar el rendimiento (Viveros-Viveros et al. 2013).

En este contexto, la construcción de modelos matemáticos o estadísticos, para predecir el área del limbo y el peso seco de las plantas es de gran importancia en el campo agrícola, porque con ellos se puede prescindir de muestreos destructivos, los cuales, implican pérdida en el material de investigación y adquisición de materiales costosos de medición. Por otro lado, se describen las etapas de desarrollo de todos los cultivos agrícolas utilizando la escala $\mathrm{BBCH}$ (Biologische Bundesanstalt Bundessortenamt Chemische Industrie), para identificar las etapas fenológicas en plantas leñosas. Este sistema está básicamente conformado por un código de dos dígitos, donde el primero define las etapas principales con valores de 0 a 9 y, el segundo dígito, identifica el estado secundario de desarrollo (Meier et al. 2009).

La variación del área del limbo durante el crecimiento es el factor más influyente en los cambios del peso seco acumulado por las plantas (Muñoz et al. 2008; NúñezRamírez et al. 2012). El área del limbo también guarda relación con sus parámetros lineales, largo y el ancho y dicha relación puede ser descrita, mediante ecuaciones de regresión (Roberti et al. 2014; Jerez et al. 2014; Interdonato et al. 2015; Unigarro-Muñoz et al. 2015; Valbuena et al. 2016; Razquin et al. 2017).

Una medición precisa del área del limbo es esencial para comprender la interacción entre el crecimiento, el desarrollo de los cultivos y el medio ambiente. Por lo tanto, otro método simple, barato, rápido, fiable y no destructivo, que involucre, por ejemplo, el contenido relativo de agua o el peso seco del limbo para estimar el área del limbo es necesario, con el fin de facilitar los experimentos en campo (Kumbhani et al. 2017).

Este estudio tuvo como objetivo determinar las mejores ecuaciones de regresión lineal múltiple, para la predicción del peso seco de los órganos y del área del limbo del duraznero, variedad Jarillo, de la misma edad del mismo origen sexual, manejo agronómico en tres localidades de la Provincia de Pamplona-Norte de Santander, tres estadios secundarios, para estimar el área del limbo (72, 75 y 87), uno, para estimar el peso seco del limbo (72); dos estadios, para estimar el peso seco del fruto (75 y 87) y un estadio principal, para hallar el peso seco de las ramas (9), siguiendo los lineamientos de la escala $\mathrm{BBCH}$, para facilitar la toma de datos en campo.

\section{MATERIALES Y MÉTODOS}

Localización. El estudio, se realizó en tres localidades, donde se obtuvieron las muestras de los órganos vegetales, como se indica en la figura 1. La primera fue Pamplona, finca Las Delicias, vereda Chíchira, con coordenadas 07²2'43,6” N y $72^{\circ} 37^{\prime} 41,1$ ” W, perteneciente a la cuenca del Catatumbo. La altitud fue de $2170 \mathrm{msnm}$, temperatura media de $16^{\circ} \mathrm{C}$, una precipitación de $933,9 \mathrm{~mm}$ al año y régimen bimodal. El tipo de suelo fue un inceptisol, con pendiente de $25 \%$ y bosque seco montano bajo (bs-MB).

La segunda en Chitagá, finca el Recuerdo, vereda Carrillo, con coordenadas $07^{\circ} 11^{\prime} 15^{\prime \prime} \mathrm{N}$ y $72^{\circ} 39^{\prime} 7,3^{\prime \prime} \mathrm{W}$, perteneciente a la cuenca del Orinoco. La altitud fue de $1870 \mathrm{msnm}$, con temperatura media de $18^{\circ} \mathrm{C}$, precipitación de $879,5 \mathrm{~mm}$ al año. Con régimen de precipitaciones unimodal, el tipo de suelo fue inceptisol, con pendiente de $25 \%$, ubicada en el bosque húmedo montano bajo (bh- MB).

Finalmente, en la tercera en Pamplonita, finca Bella vista, vereda Batagá, con coordenadas $07^{\circ} 26^{\prime} 18,1^{\prime \prime}$ N y $72^{\circ} 38$ 9" W, perteneciente a la cuenca del Catatumbo, con una altitud de $1670 \mathrm{msnm}$, temperatura media $20^{\circ} \mathrm{C}$, precipitación de $1200 \mathrm{~mm}$ al año, en un régimen bimodal. El suelo fue un inceptisol, con pendiente de $25 \%$, ubicada en el bosque seco montano bajo (bs- MB) (IGAC, 2006).

Material vegetal. La variedad Jarillo es de bajo requerimiento de frío, es decir, necesita para entrar en floración, menos de 400 horas frío acumuladas durante su reposo; presenta árboles francos provenientes de semilla sexual de gran tamaño, debido a la distancia de plantación de 6 a $7 \mathrm{~m}$ entre plantas y surcos, los cuales, fueron escogidos en cada 


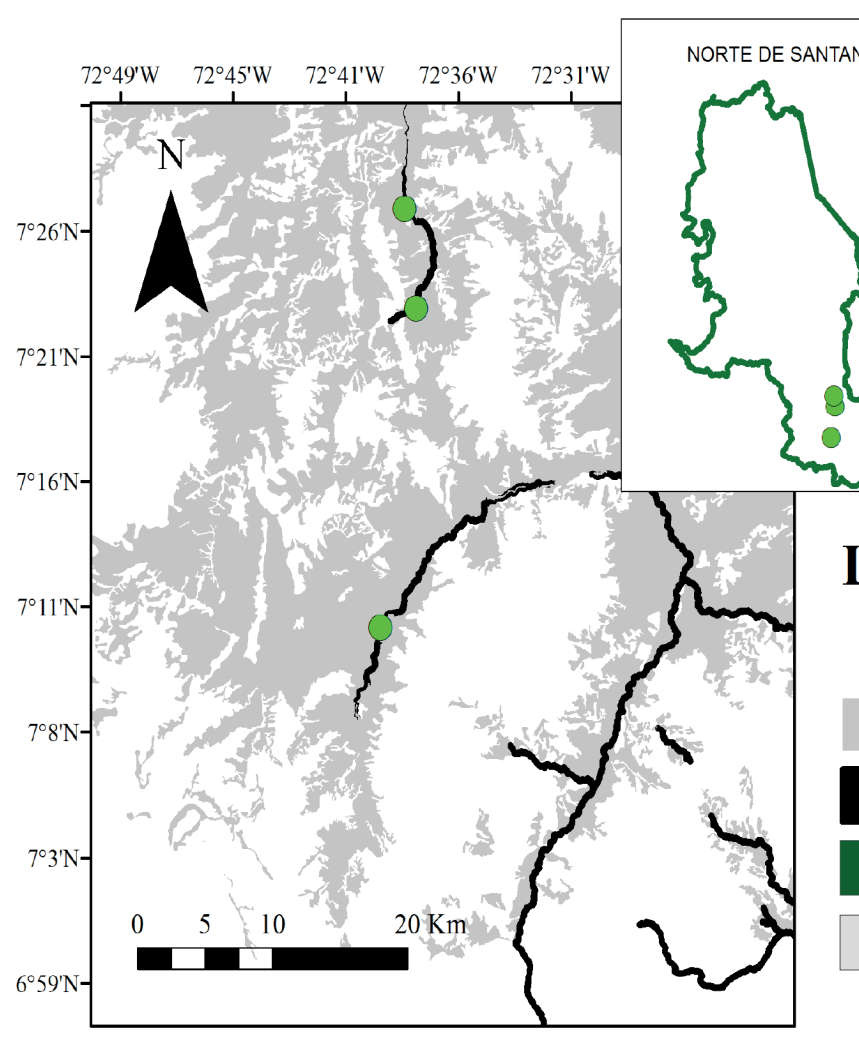

\section{Leyenda}

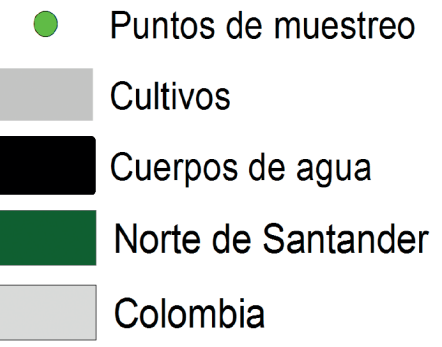

Elaboró: B.Sc., Carlos H. Cáceres-Martínez

Figura 1. Ubicación de las localidades de muestreo del duraznero, variedad Jarillo, en tres diferentes altitudes. En la parte superior Pamplonita $(1.670 \mathrm{msnm})$, en el medio Pamplona $(2.170 \mathrm{msnm})$, ambas a orillas del río Pamplonita (Cuenca del Catatumbo) y en la parte inferior Chitagá (1.870msnm), a orillas del rio Chitagá (Cuenca del Orinoco).

localidad, según las condiciones de pendiente de $25 \%$ y manejo agronómico forzado, usado en el trópico alto, que incluyó el mismo tipo de raleo de frutos en el estadio 72 de endurecimiento del endocarpio y podas de fructificación, al inicio de cada ciclo productivo. Se conformaron parcelas con árboles en plena producción agronómica y con edad mayor a los diez años, según lo describen Avilán et al. (2009) y Campos (2013).

Muestreo. La colecta del material vegetal, se realizó en la franja altitudinal ubicada entre 1.670 y $2.170 \mathrm{msnm}$, donde se ubican la mayoría de los cultivos de duraznero, de la provincia de Pamplona; se fijó el muestreo por localidad en cultivos, que tenían la misma pendiente, la misma edad en los árboles, el mismo origen sexual, el mismo estadio de desarrollo logrado con el manejo forzado, igual tipo de poda y raleo, durante el ciclo productivo.
De los árboles, se tomaron 10 frutos de apariencia similar en color y en tamaño, cosechados de la parte media de la rama mixta, en dos estadios secundarios de desarrollo, a los 72 dddd (días después de la defoliación en el estadio secundario 75) y a los 172 dddd (87); estas ramas son las que presentan mayor cantidad de yemas reproductivas y estaban ubicadas en el tercio medio. En 10 hojas, ubicadas en el tercio medio del árbol en la parte expuesta al sol y 10 ramas mixtas del tercio medio, se les midió las variables regresoras, para estimar el peso seco de frutos, limbo, rama mixta y el área del limbo.

Para el muestreo de árboles en cada localidad, se utilizó el método del hipercubo latino condicional, propuesto por Minasny et al. (2012), una red rígida, donde se identificaron, primero, 48 árboles, ubicados en 6 filas y 8 columnas, incorporando, como variable auxiliar, el área del involucro foliar del árbol de forma elíptica, con la relación asociada 
al área de una elipse, $\pi a b$, donde $a$, corresponde al semieje mayor (largo en metros) y $b$, al semi-eje menor (ancho en metros). Con la ayuda de la librería clhs del software R, se generó una muestra de 8 árboles por localidad. Además, también formaron parte de las variables auxiliares del muestreo condicional, el largo (eje mayor en $\mathrm{cm}$ ) y ancho (eje mayor en $\mathrm{cm}$ ), del limbo, su producto $\left(\mathrm{cm}^{2}\right)$, el peso seco del limbo $(\mathrm{g})$, el área del limbo $\left(\mathrm{cm}^{2}\right)$, el diámetro longitudinal del fruto $(\mathrm{cm})$, el muestreo que corresponde al día que se recolectaron las muestras vegetales, la altitud o la localidad $(\mathrm{m})$, la longitud de la rama mixta $(\mathrm{cm})$ ubicada en el estrato medio del árbol, la relación diámetro basal/medio de la rama, el número de yemas por ramas mixtas y el volumen de la rama mixta $\left(\mathrm{cm}^{3}\right)$.

Los muestreos, se realizaron en los siguientes estadios fenológicos, utilizando la escala $\mathrm{BBCH}$ : la etapa secundaria 72: ovario verde rodeado de corona de pétalos que mueren, que corresponde al inicio del crecimiento del fruto; la etapa secundaria 75: fruto de aproximadamente la mitad del tamaño final y es cuando se presenta el endurecimiento del endocarpio y, en la etapa secundaria 87: fruto maduro para la cosecha y 9: senescencia y comienzo del reposo (Mounzer et al. 2008).

El área del limbo fue determinada en 240 hojas maduras del tercio medio del árbol, del tercio medio de la rama mixta y cercana al fruto en formación, con 2,5 meses de edad, aproximadamente, en las tres localidades, mediante el uso de ImageJ, que es un programa de procesamiento de imagen digital, de dominio público, programado en Java y desarrollado en el National Institute of Health, de Estados Unidos. El proceso empezó con la descarga de la primera foto de la hoja completa de duraznero recogida en campo, en junio de 2014 y la inclusión del largo del limbo, como parámetro de entrada de esa misma hoja, el cual, se midió, previamente, con un pie de rey electrónico, marca Ubermann; con estos parámetros, el software estimó el área del limbo. Además, se midió el ancho (ANL) y largo del limbo (LONL) y obtuvo su producto, el diámetro longitudinal fruto (DLF), que fue el mayor al principio del crecimiento del fruto, el diámetro basal (DB), el diámetro medio (DM) y el volumen de rama mixta (VR); para hallar el volumen de las ramas, se aplicó la fórmula del cono truncado, longitud de ramas mixtas (LR), diámetro basal/medio (DB/DM).

El peso seco, se midió después de secados los limbos, las ramas mixtas y los frutos. Se usó el método tradicional de secado de muestras, para la determinación de peso seco, que se realiza mediante el uso de una estufa de circulación forzada, a $65^{\circ} \mathrm{C}$, durante un lapso que varió entre 24 a 72 horas, dependiendo del tipo de muestra del órgano a analizar, empleando un horno eléctrico de secado de circulación forzada de aire caliente seco, marca Mettler
Toledo, de fabricación estadounidense. El peso seco, se obtuvo mediante una balanza electrónica, marca Lexus, de fabricación China, con aproximación de dos dígitos, rango de medida $3.600 \mathrm{~g}$ y precisión de $0,01 \mathrm{~g}$. Se contó el número de yemas vegetativas y reproductivas por rama mixta, recolectada en el tercio medio del árbol. Se tuvo en cuenta, como variable regresora, el muestreo y la altitud, para estimar el peso seco del fruto.

Por último, los muestreos conformaron una matriz de 240 observaciones, discriminadas en 80 , por localidad. Las variables medidas, se asociaron a la medición del peso seco de 80 frutos, al peso seco y al área de 80 limbos y al peso seco de 80 ramas mixtas, por localidad. Para recolectar la información, se tuvo en cuenta las tres etapas de crecimiento para limbos (72, 75 y 87), dos para frutos (75 y 87) y uno para ramas mixtas (9), para un total de 240 limbos, por estadio, en el 72, 75 y 87, ubicadas en el tercio medio del árbol; 240 frutos por muestreo, en el estadio 75 y en el estadio 87, ubicados en el tercio medio de la rama mixta y para 240 ramas mixtas, ubicadas en el tercio medio del árbol, en el periodo de reposo del cultivo (Estadio principal 9).

Variables regresoras. En el estudio, se involucró una matriz de datos construida con la información obtenida en los siguientes estadios secundarios de desarrollo: inicio de crecimiento del fruto (72), endurecimiento del endocarpio (75), inicio de la madurez del fruto (87) y uno principal, correspondiente al estadio de reposo del cultivo (9), en la variedad jarillo, para el cual, se dispuso de diferentes variables del órgano a analizar. Para hallar el área del limbo (AL), se usó: el ancho del limbo (ANL), el largo del limbo (LONL), el producto de LONL x ANL y el peso seco del limbo (PSL); para el peso seco del limbo (PSL): ancho del limbo (ANL) y largo del limbo (LONL); para hallar el peso seco del fruto (PSF): largo del limbo (LONL), diámetro longitudinal del fruto (DLF), área del limbo (AL); MU: muestreo por estadio de desarrollo y ALT altitud o localidad y para el peso seco de la rama (PSR): número de yemas (NY), volumen de la rama mixta (VR), longitud de la rama mixta (LR) y diámetro basal de la rama /diámetro medio de la rama (DB/DIM).

Análisis estadístico. Se escogieron los mejores modelos de regresión lineal múltiple, utilizando el paquete estadístico SPPS 23, con el método de selección de variables Stepwise, donde se introdujeron las variables regresoras y las respuestas, para estimar el área y el peso seco del limbo, de los frutos y de las ramas mixtas, teniendo en cuenta que el $\mathrm{R}^{2}$ fuera, al menos, de 0,7, que es el estadístico que representa la proporción de variación explicada por la regresión; el $\mathrm{R}^{2}$ ajustado, la significancia del modelo en su anava, la suma de cuadrados del error, el cuadrado medio del error experimental y la significancia de los parámetros de la regresión. Con respecto a la validación de los modelos de regresión, se 
tuvieron en cuenta los siguientes supuestos, que son: linealidad, independencia, homocedasticidad, normalidad y no-colinealidad. Por último, se realizó comparación de los datos observados con los estimados, para observar la relación entre ellos, por medio de una regresión lineal simple, para la rama y el limbo. La regresión para el fruto fue de tipo cuadrático.

\section{RESULTADOS Y DISCUSIÓN}

Se muestra las ecuaciones halladas, para estimar el área y el peso seco del limbo, del fruto y de la rama mixta y la regresión entre los datos observados y los estimados, para el área del limbo y los pesos secos de cada órgano. Según la tabla 1, el mejor modelo para estimar el área del limbo, en condiciones
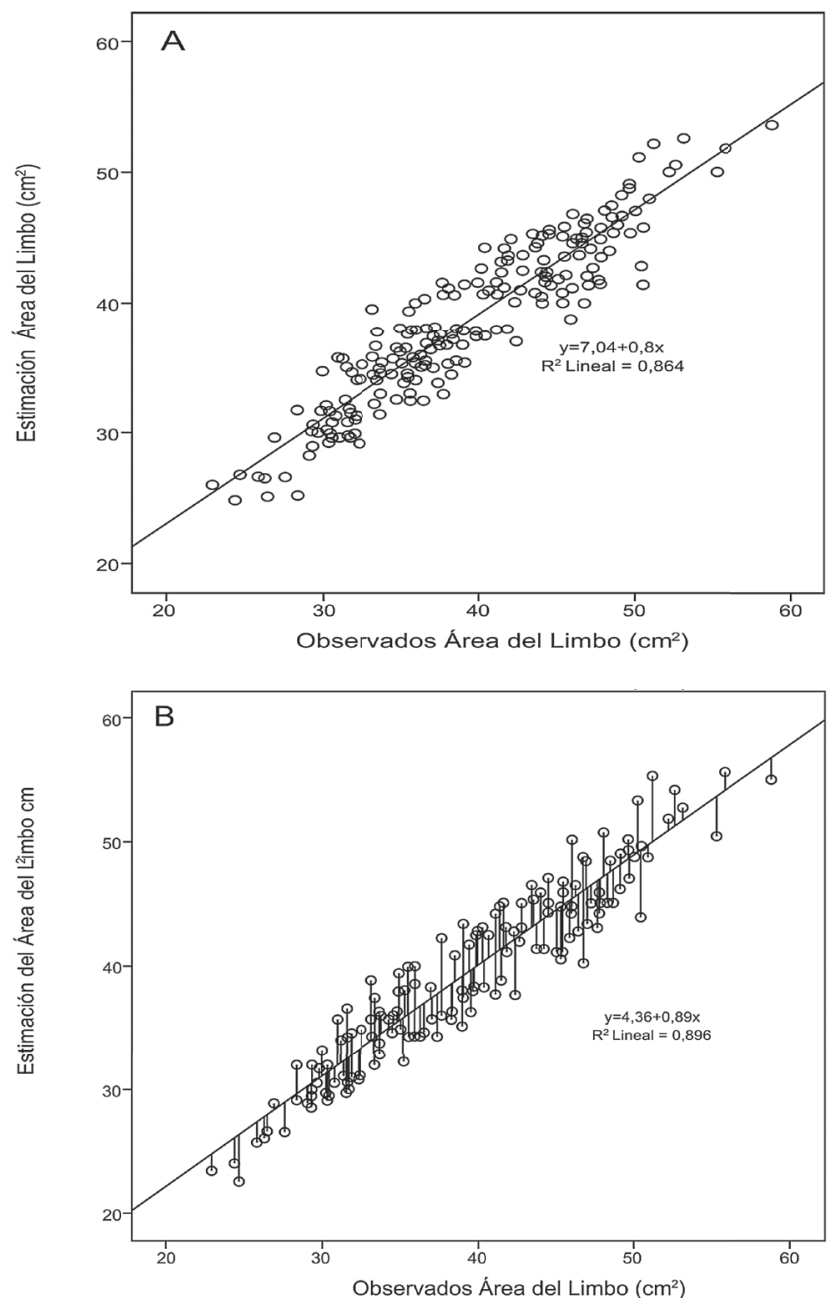

de las localidades al inicio del crecimiento del fruto, fue $\mathrm{AL}=-4,998+0,294$ (LONLxANL) + 4,541ANL+29,564 PSL, con un $\mathrm{R}^{2}=0,861$ y un comportamiento normal de los datos, cumpliendo con los supuestos establecidos, para este modelo de regresión. En la figura $2 \mathrm{~A}$, se muestra la estrecha relación lineal que se presenta entre los datos estimados con los datos observados e indica que se puede predecir el área del limbo $\mathrm{y}$, con ella, se obtuvo un $\mathrm{R}^{2}=0,864$, tal como lo muestra, en forma similar, el trabajo realizado por Goharnejad et al. (2014). Para la relación entre el área del limbo observada y estimada, mediante distintos métodos, por Razquin et al. (2017), obtuvieron que y $=465+0,96 x \mathrm{y}$ un $\mathrm{R}^{2}=0,80$ valor que se encuentra por debajo del hallado en este estudio.

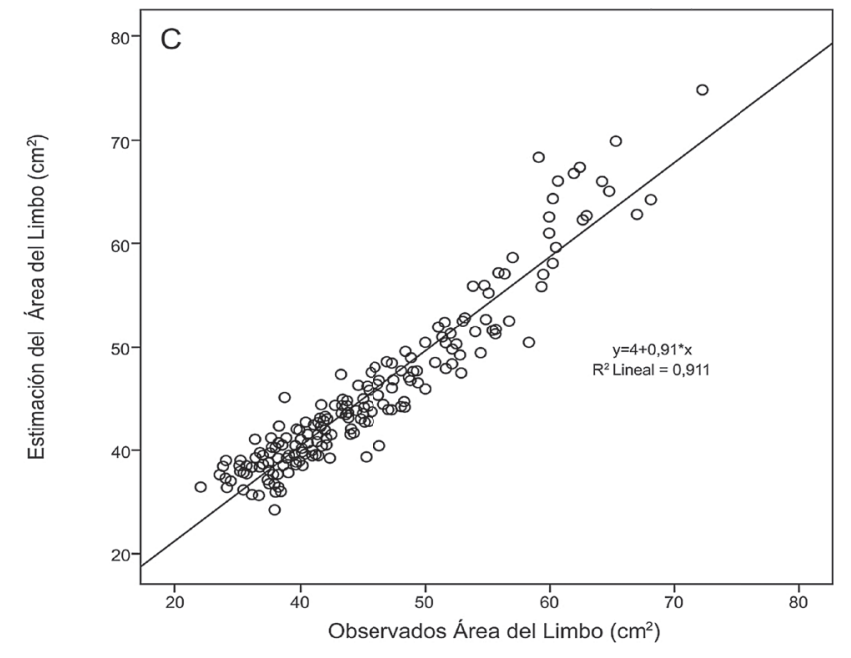

Figura 2. Relación entre los datos predichos y observados, obtenidos de la variable fisiológica área foliar del limbo, para las tres localidades: A) En el estadio 72 (inicio del crecimiento del fruto); B) En el estadio secundario 75 (endurecimiento del endocarpio) y C) En el estadio secundario 87 (Madurez del fruto) del duraznero, variedad Jarillo. 


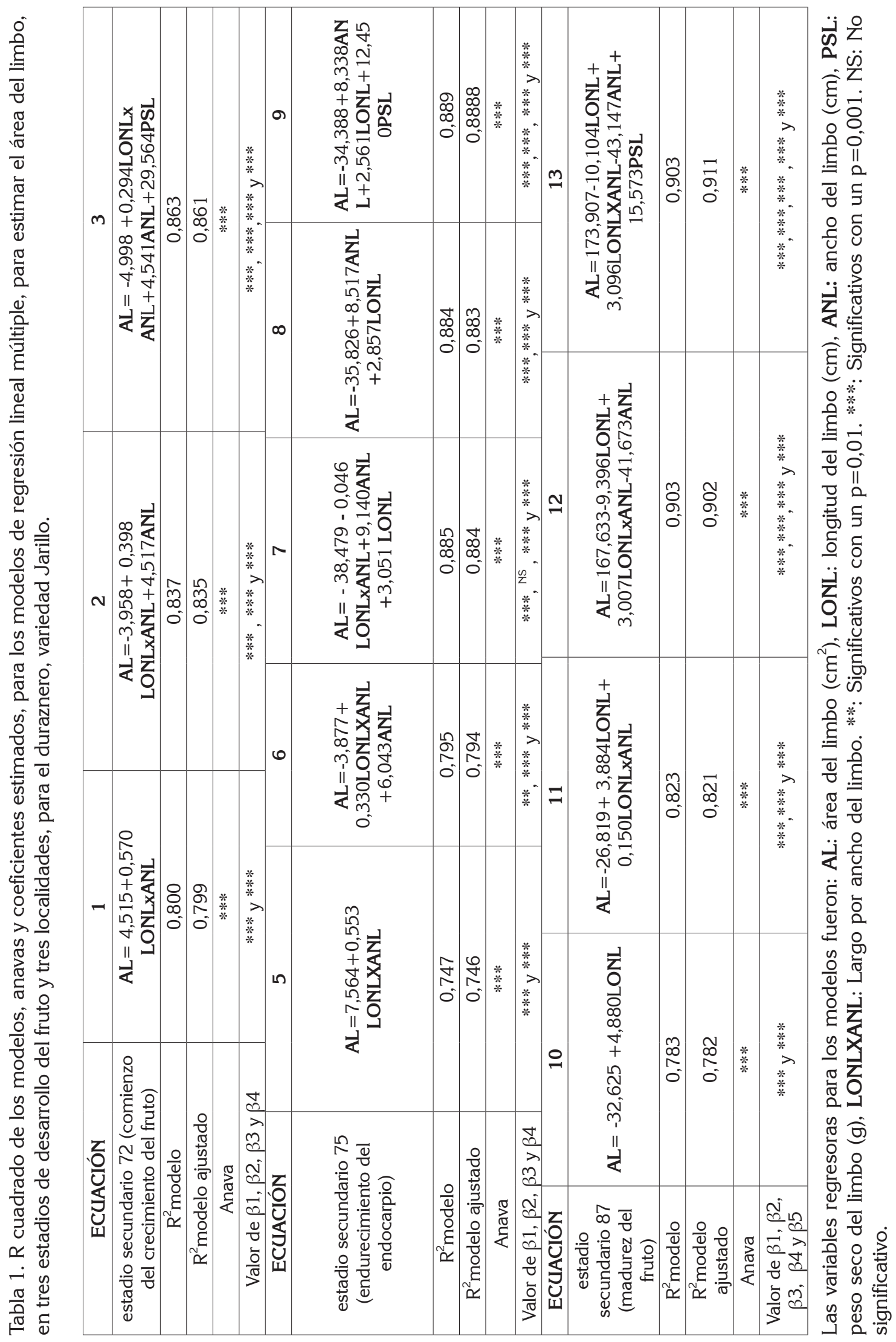


El peso seco del limbo se incluyó en la ecuación y se puede tomar como parámetro para el desarrollo de modelos lineales, para estimar el área del limbo en plantas, tal como lo muestra en su trabajo Kumbhani et al. (2017) y las dimensiones lineales del limbo, como lo describen Quevedo et al. (2012), Unigarro-Muñoz et al. (2015) y Buttaro et al. (2015). Valbuena et al. (2016) estimaron el área del limbo en $B$. brizanta usando la ecuación con variables regresoras lineales asociadas al limbo, de la siguiente manera: bo+b3 $\mathrm{X} 3$, donde, bo $=$ constante y b3 X3=largo por ancho de las hojas y obtuvieron un coeficiente de regresión de 0,67 , valor que se encuentra por debajo del hallado en este estudio.

Según el anava para la regresión de la tabla 1, el mejor modelo que se ajustó en el momento del endurecimiento del endocarpio del fruto fue la ecuación $A L=$ 35,826+8,517ANL+2,857LONL. Esta ecuación utiliza, para estimar el área del limbo, solo dimensiones lineales, lo que le da ventajas para su medición en campo y por su bajo costo, tal como lo demuestran trabajos desarrollados por Pandey \& Singh (2011), Unigarro-Muñoz et al. 2015, Tondjo et al. (2015) y Casierra-Posada et al. (2017). Los datos recabados presentaron una distribución normal, validando los supuestos del modelo y un $\mathrm{R}^{2}=0,884$. En la figura $2 \mathrm{~B}$, se puede observar la relación estrecha que hay entre los datos estimados y los observados, que tuvieron un ajuste de $\mathrm{R}^{2}=0,896$; esto indicó, que la estimación del área del limbo es significante, para esta ecuación, en el endurecimiento del endocarpio.

Las variables independientes ancho del limbo y el largo del limbo fueron las que mejor se comportaron, para estimar el área del limbo. Otro trabajo similar sobre hojas lanceoladas, como la del duraznero, fue desarrollado por Jerez et al. (2014). Según los resultados del anava que se muestran la tabla 1, el mejor modelo que se ajustó al momento de la cosecha, para estimar el área del limbo, fue el representado por la ecuación $\quad A L=173,907-10,104 L O N L+3,096$ (LONLxANL)43,147ANL+15,573PSL.

Los datos conseguidos tuvieron un comportamiento normal y un $R^{2}=0,914$. En la figura $2 C$, se muestra la relación que tienen los datos observados con los estimados del área del limbo, formando una línea recta y con un ajuste de $R^{2}=0,911$. Este resultado es similar a los resultados obtenidos por Burgos et al. (2010) y Goharnejad et al. (2014), quienes encontraron que, para el cultivo de yuca, el área foliar puede ser estimada usando simples mediciones lineales, tales como la longitud del pecíolo, la sumatoria de la longitud de los lóbulos de la hoja palmeada y la superficie del lóbulo central. Las variables independientes largo del limbo, el producto largo por ancho del limbo, ancho del limbo y el peso seco del limbo son las que mejor sirvieron, para estimar el área del limbo.
Por otro lado, la distribución homogénea evidencia una baja dispersión de los datos observados respecto a los estimados en este estudio y este modelo permite valorar el área foliar en este estado del ciclo del cultivo, tal como lo afirman Interdonato et al. (2015).

Según los resultados que arroja el anava, presente en la tabla 2, el mejor modelo que se ajustó, para estimar el peso seco del limbo en el momento del inicio de la formación del fruto, fue el representado por la ecuación $\mathrm{PSL}=0,022+0,005 \mathrm{AL}+0,006 \mathrm{LONL}$. Los datos recabados tuvieron un comportamiento normal, cumpliendo con todos los supuestos de validación del modelo y un $R^{2}=0,576$, que es inferior a 0,7 y no permite estimar esta variable fisiológica. La figura 3A muestra la relación lineal que tienen los datos observados con los estimados, donde se ajustan con un $\mathrm{R}^{2}=0,597$ e indicando que, con ésta, no se puede estimar el peso seco del limbo del duraznero, variedad Jarillo. Los resultados fueron similares a los obtenidos por Quevedo et al. (2012), quienes realizaron una figura similar a la figura $3 \mathrm{~A}$, donde graficaron la relación lineal entre los datos estimados y los observados, a partir de la ecuación del peso seco del limbo. Las variables independientes área y largo del limbo son útiles, para estimar el peso seco del limbo en el duraznero, variedad Jarillo, concordando el uso de estas variables regresoras con estudios adelantados por Çırak et al. (2005) y Roberti et al. (2014), pese que, al ser menor a 0,7 el $R^{2}$, el modelo hallado no permite estimar el peso seco del limbo.

Según el anava, de la tabla 2, el mejor modelo que se ajustó en las condiciones evaluadas en el endurecimiento del endocarpio del fruto y en la cosecha del fruto, para estimar el peso seco del fruto, fue $\mathrm{PSF}=62,985$ 10,452MU+10,232DLF-4,231ALT-0,663AL+2,067LONL, con un $R^{2}=0,8218$. En la figura $3 B$, se muestra una tendencia curvilínea de los datos estimados de la materia seca del fruto respecto a los observados, que se ajusta a un $R^{2}=0,869$.

Los datos registrados no tuvieron un comportamiento normal violando, así, este supuesto. Se transformaron las variables para que siguieran la tendencia de la campana de Gauss, pero al final, se dejaron los datos con comportamiento no normal. Esta falta de normalidad se debe, probablemente, a la naturaleza misma de la variable respuesta que tomó, por tratarse de frutos de dimensiones variables por localidades. La inclusión en la ecuación de regresión de la altitud, como variable regresora, supone que las localidades se comportan en forma diferencial ante la respuesta presentada por el peso seco del fruto, como lo hallado en el análisis discriminante por Quevedo et al. (2017), al manejar variables morfológicas asociadas al fruto e, indicaría, que el manejo de los datos de esta variable fisiológica debe ser por localidad y no 

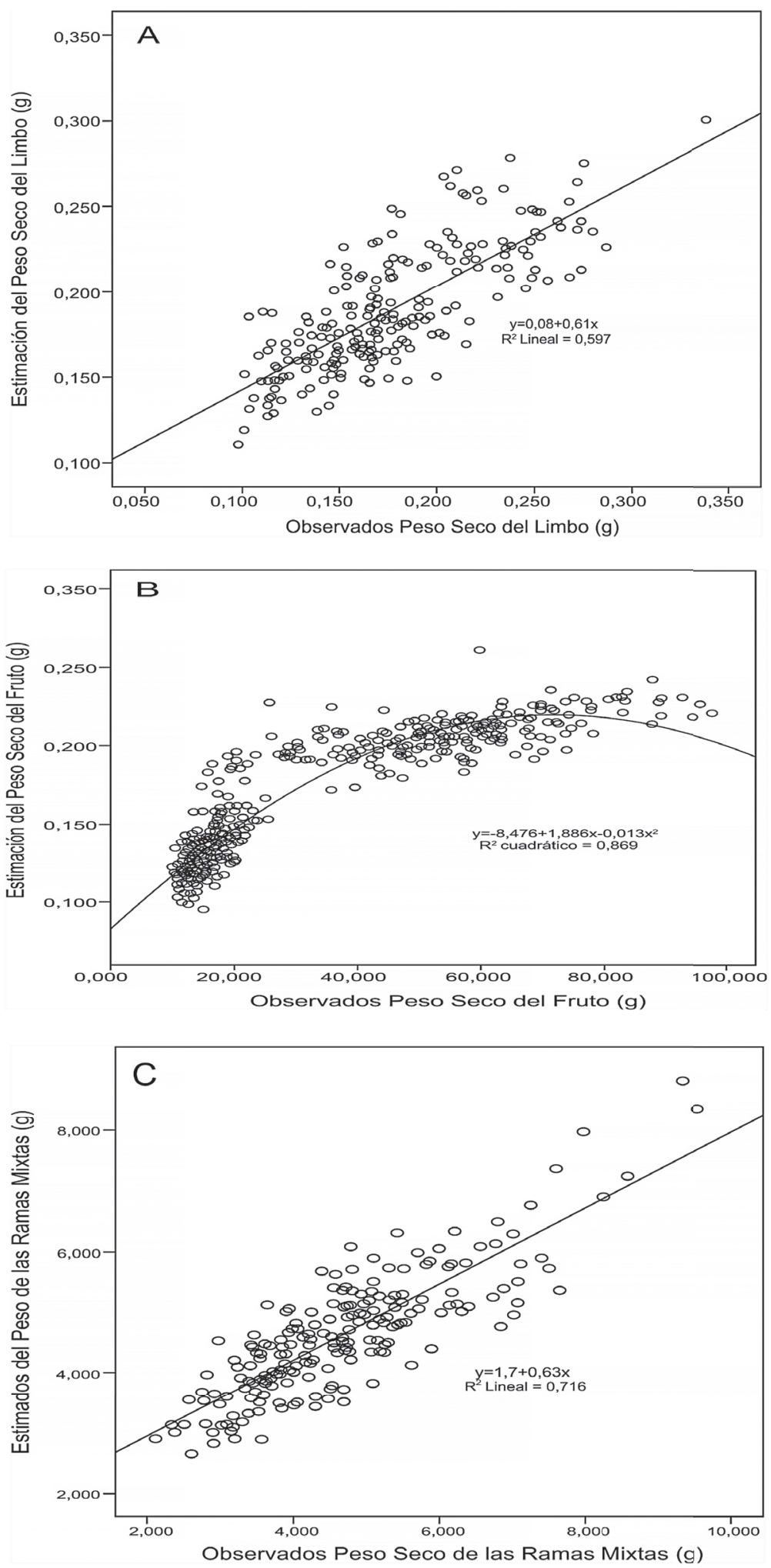

Figura 3. Relación entre los datos predichos y observados, obtenidos del carácter fisiológico: A) Peso seco del limbo, para las tres localidades, en el estadio secundario 72 (al inicio del crecimiento del fruto); B) Peso seco del fruto, en los estadios secundarios 75 (durante el endurecimiento del endocarpio) y 87 (durante la madurez del fruto) y C) Peso seco de las ramas en el estadio 9 (Estadio de reposo del cultivo), del duraznero, variedad Jarillo. 


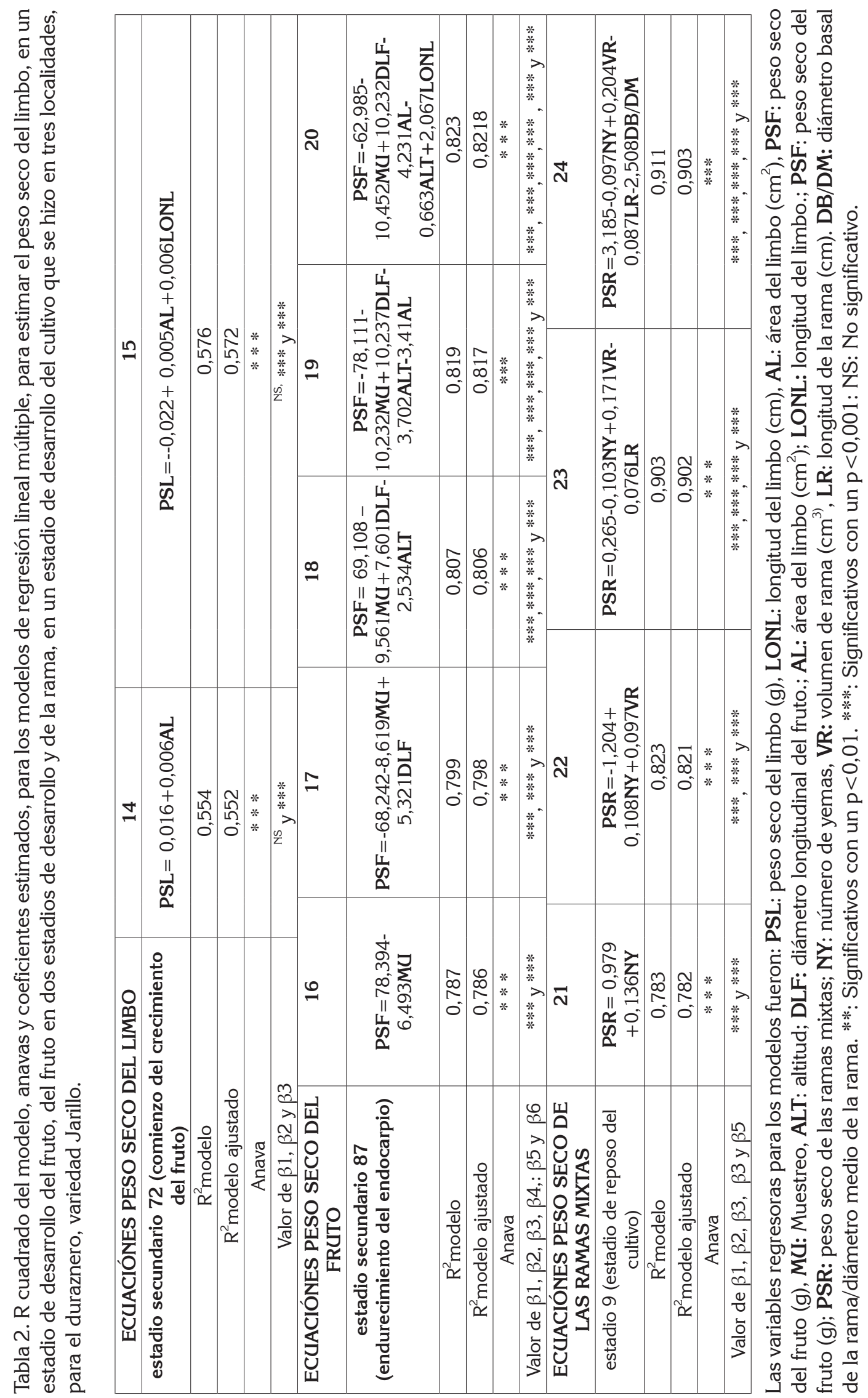


combinado de las tres y por estadio fenológico, sin combinar varios de ellos, porque aumenta la variabilidad.

Según los resultados del anava, que se muestran en la tabla 2 , el mejor modelo que estimó el peso seco de la rama mixta durante el estadio principal de reposo del cultivo fue $\mathrm{PSR}=3,185+0,097 \mathrm{NY}+0,204 \mathrm{VR}-0,087 \mathrm{LR}-2,508 \mathrm{DB} / \mathrm{DM}$, que proporciona evidencia para afirmar que el número de yemas, el volumen, la longitud y la relación diámetro basal/ medio de las ramas mixtas, permiten estimar el peso seco de las mismas; el modelo escogido en este estadio de desarrollo del cultivo tenía un coeficiente determinación ajustado de $R^{2}=0,903$. En la figura $3 \mathrm{C}$, se muestra que los datos observados, respecto a los estimados del peso seco de las ramas mixtas, se encuentran agrupados linealmente y que estiman el peso seco de las ramas mixtas, en condiciones del estudio, con un ajuste del $\mathrm{R}^{2}$, del 0,716 , que corresponde al estadio de reposo del cultivo e, indicaría, que es recomendable recolectar los datos de las variables regresoras en ese momento, para estimar el peso seco de las ramas, debido al mejor ajuste que se obtuvo.

Barrero et al. (2015) utilizó la regresión logarítmica, para estimar el peso seco de ramas, de una especie de uso forestal, encontrando en su trabajo coeficientes de determinación y determinación ajustado, de 0,944 y 0,943, respectivamente, algo mayor al encontrado en este estudio de coeficientes de determinación y determinación ajustado, de 0,911 y 0,903, respectivamente.

Por otro lado, Ferrando et al., en 2000/2001, hallaron en su trabajo, una ecuación para estimar el peso seco de las ramas mixtas, usando la ecuación $y=3,276+0,788 x$, con un $\mathrm{R}^{2}=0,85$ y un $\mathrm{p}<0,01$, donde $\mathrm{X}=\mathrm{DAP}^{2}$ (diámetro a la altura del pecho en $\mathrm{cm}$ ) $* \mathrm{H}=$ altura del árbol en $\mathrm{m}$.

Con los modelos electos, se pudo estimar el área del limbo, el peso seco del limbo, fruto y ramas mixtas, en las condiciones evaluadas por estado de desarrollo, debido a que los datos se comportaron de una manera normal y no fue necesario hacer trasformación de variables, con excepción de la ecuación escogida, para estimar el peso seco del fruto. Además, cumplen con los supuestos de validación del modelo y tiene significancia en la suma de cuadrados, suma de cuadrados ajustados y valores bajos en sus cuadrados medios del error.

Graficando los valores observados contra los estimados en cada uno de los escenarios planteados para cada ecuación existió una relación significativa, con tendencia cuadrática, en el caso del peso seco del fruto y ajuste del $\mathrm{R}^{2}$, por encima del 0,7, con excepción del peso seco del limbo. De acuerdo con lo anterior, se puede concluir, que la estimación del área del limbo puede ser predicha con base en la ecuación de regresión, hallada al inicio del crecimiento del fruto $A L=-$
4,998+0,294(LONLxANL)+4,541ANL+29,564PSL; por esta ecuación, puede ser explicado el $86,1 \%$ de la variabilidad total existente en el limbo.

Durante el estadio del endurecimiento del endocarpio $\mathrm{AL}=35,826+8,517 \mathrm{ANL}+2,857 \mathrm{LONL}$; por esta ecuación, puede ser explicado el $88,3 \%$ de la variabilidad total existente en el área del limbo. El mejor modelo que se ajustó para estimar el área del limbo durante la cosecha del fruto fue $A L=173,907-10,104 \mathrm{LONL}+3,096(\mathrm{LONLxAN})$ L-43,147ANL+15,573PSL; por esta ecuación, puede ser explicado el 90,03\% de la variabilidad total existente en el área del limbo.

El peso seco del limbo no puede ser estimado satisfactoriamente en el inicio del crecimiento del fruto, por PSL $=0,022+0,005 \mathrm{AL}+0,006 \mathrm{LONL}$, porque con esta ecuación, apenas puede ser explicado el $57,2 \%$ de la variabilidad total existente en el peso seco del limbo. El peso seco del fruto puede ser estimado satisfactoriamente en endurecimiento del endocarpio y cosecha, por $\mathrm{PSF}=62,985$ 10,452MU+10,232DLF-4,231ALT-0,663AL+2,067LONL; por esta ecuación, puede ser explicado el $82,18 \%$ de la variabilidad total existente en el peso seco del fruto.

El peso seco de las ramas puede ser estimado satisfactoriamente, por $\mathrm{PSR}=3,185+0,097 \mathrm{NY}+0,204 \mathrm{VR}$ 0,087LR-2,508DB/DM y por esta ecuación, puede ser explicado el $91,10 \%$ de la variabilidad total existente en el peso seco de las ramas mixtas.

Lo anterior indica que las variables regresoras número de yemas, volumen de las ramas, longitud de las ramas mixtas y el diámetro basal/medio, peso seco del fruto, muestreo, diámetro longitudinal del fruto, altitud o localidad, largo del limbo, peso seco del limbo, área del limbo, el producto del largo por el ancho del limbo, ancho del limbo, peso seco del limbo pueden ser usadas según sea el caso a analizar, para estimar el peso seco de los órganos y el área foliar de $P$. persica (L.) (Batsch), variedad Jarillo.

Conflictos de intereses: El manuscrito fue preparado y revisado con la participación de todos los autores, quienes declaramos que no existe conflicto de intereses que ponga en riesgo la validez de los resultados presentados. Financiación: Este estudio fue financiado por la Universidad de Pamplona, los productores Carlos Alberto Portilla y William Eduardo Flórez y los autores.

\section{BIBLIOGRAFÍA}

1. AVILÁN, L.; SOTO, E.; PÉREZ, M.; MARÍN, C.; RODRÍGUEZ, M.; RUIZ, J. 2009. Comportamiento fenológico de la raza antillana de aguacate en la 
región centro-norte costera de Venezuela. Agron. Trop. (Venezuela). 59(1):5-14.

2. BARRERO, M.H.; ARGUELLE, W.T.; BRAVO-IGLESIAS, J.A.; VIDAL, C.A.; AJETE, H.A.; CASTILLO-EDUA, B.R. 2015. Estimación de la biomasa de ramas secas en plantaciones de Pinus maestrensis Bisse de la provincia Granma, Cuba. Rev. Cubana de Ciencias Forestales, Vol. 3(1). Disponible desde Internet en: https://dialnet.unirioja.es/ ejemplar/ 407237.pdf (con acceso 01/08/2017).

3. BURGOS, A.M.; AVANZA, M.M.; BALBI, C.N.; PRAUSE, J; ARGÜELLO, J.A. 2010. Modelos para la estimación no destructiva del área foliar de dos cultivares de mandioca (Manihot esculenta Krantz) en la Argentina. Agriscientia (Argentina). 27(1):55-61.

4. BUTTARO, D.; ROUPHAEL, Y.; RIVERA, C.M.; COLLA, G; GONNELLA, M. 2015. Simple and accurate allometric model for leaf area estimation in Vitis vinifera L. genotypes. Photosynthetica (República Checa). 53(3):342-348.

5. CAMPOS, T.DEJ. 2013. Capítulo 4: Especies y variedades de hoja caduca en Colombia. In: Miranda, D.; Fischer, G.; Carranza, C. Los frutales caducifolios en Colombia - situación actual, sistemas de cultivo y plan de desarrollo, Sociedad Colombiana de Ciencias Hortícolas, Bogotá. p.47-64.

6. CASIERRA-POSADA, F.; ZAPATA-CASIERRA, V.; CUTLER, J. 2017. Comparación de métodos directos e indirectos para la estimación del área foliar en duraznero (P. persica) y ciruelo (Prunus salicina). Rev. Colomb. Cienc. Hortic. (Colombia). 11(1):3038.

7. CARRANZA, C.; MIRANDA, D.; FISCHER, G. 2013. Capítulo 5: Zonificación actual de los sistemas de producción de frutales caducifolios en Colombia. In: Miranda, D.; Fischer, G.; Carranza, C. Los frutales caducifolios en Colombia - situación actual, sistemas de cultivo y plan de desarrollo, Sociedad Colombiana de Ciencias Hortícolas, Bogotá. p.67-86.

8. ÇIRAK, C.; ODABAŞ, M.S.; SAĞLAM, B.; AYAN, A.K. 2005. Relation between leaf area and dimensions of selected medicinal plants. Res. Agr. Eng. (Turquía). 51(1):13-19.

9. FERRANDO, J.J.; GOYA, J.F.; BARRERA, M.D.; YAPURA, P.F.; FRANGI, J.L. 2000/2001. Biomasa y productividad de bosques de Austrocedrus chilensis en rio Negro Argentina. Rev. Fac. Agron. La Plata (Argentina). 104(2):139-149.

10. FISCHER, G. 2005. Aspectos de la fisiología aplicada de los frutales promisorios en cultivo y poscosecha. Rev. Comalfi (Colombia) 32(1):22-34.

11. FISCHER, G.; ALMANZA-MERCHÁN, P.J.; RAMÍREZ, F. 2012. Source-sink relationships in fruit species. A review. Rev. Colomb. Cienc. Hortic. 6 (2):238-253.

12. GOHARNEJAD, A.; ZAREI, A.; TAHMASEBI, P. 2014. Comparing multiple regression, principal componant analysis, partial least square regression and ridge regression in predicting rangeland biomass in the semi steppe rangeland of Iran. Environment and Natural Resources J. (Tailandia). 12(1):1-21.

13. INSTITUTO GEOGRÁFICO AGUSTÍN CODAZZI -IGAC-. 2006. Estudio general de suelos y zonificación de tierras: del departamento de Norte de Santander. 2. ${ }^{2}$ Edición. Bogotá, IGAC, Colombia. 359p.

14. INTERDONATO, R.; ROMERO, J.I; BAS NAHAS, S.S.; ROBERTI, J.O.; RODRÍGUEZ R., J.A.; ROMERO, E.R. 2015. Estimación no destructiva del área foliar por planta en sorgos bioenergéticos. Rev. Agron. Noroeste Argent. (Argentina). 35(1):51-53.

15. JEREZ, M.E.; MARTÍN, M.R.; DÍAZ, H.Y. 2014. Estimación de la superficie foliar en dos variedades de papa (Solanum tuberosum L.) por métodos no destructivos. Comunicación corta. Cultivos Tropicales (Cuba). 35(1):57-61.

16. KUMBHANI, N.R.; KUVAD, R.P.; THAKER, V.S. 2017. Development of linear model for leaf area measurement of two medicinally important plants: Helicteres isora L. and Vitex negundo L. J. App. Biol. Biotech. (India). 5(03):057-060.

17. MEIER, U.; BLEIHOLDER, H.; BUHR, L.; FELLER, C.; HACK, H.; HEß, M.; LANCASHIRE, P.D.; SCHNOCK, U.; STAUß, R.; VAN DEN BOOM, T.; WEBER, E.; ZWERGER, P. 2009. The BBCH system to coding the phenological growth stages of plants - history and publications. J. für Kulturpflanzen. 61(2):41-52.

18. MINASNY, B.; MALONE, B.P.; MCBRATNEY, A.B. 2012. Digital soil assessment and beyond. CRC Press, Taylor \& Francis Group, London. 482p.

19. MOUNZER, O.H.; CONEJERO, W.; NICOLA'S, E.; ABRISQUETA, I.; GARCÍA-ORELLANA, Y.V.; TAPIA, 
L.M.; VERA, J.; ABRISQUETA, J.M.; RUIZ-SÁNCHEZ, M.DELC. 2008. Growth pattern and phenological stages of early-maturing peach trees under a Mediterranean climate. HortScience (Estados Unidos). 43(6):1813-1818.

20. MUÑOZ, F.; ESPINOSA, M.; CANCINO, J.; RUBILAR, R.; HERRERA, M. 2008. Efecto de poda y raleo en el área foliar de Eucalyptus nitens. Bosque (Chile). 29(1):44-51.

21. NÚINEZEZ-RAMÍREZ, F.; GRIJALVA-CONTRERAS, R.L.; MACÍAS-DUARTE, R.; ROBLES-CONTRERAS, F.; CECEÑA-DURÁN, C. 2012. Crecimiento, acumulación y distribución de materia seca en tomate de invernadero. BIOtecnia (México). 14(3):25-31.

22. PANDEY, S.K.; SINGH, H. 2011. A simple, cost-effective method for leaf area estimation. J. of Botany (India) 2011: Disponible desde Internet en: https://www. hindawi.com/journals/jb/2011/658240/ (con acceso 1/08/2017).

23. PINZÓN, E.H.; CRUZ, M.A.; FISCHER G. 2014. Aspectos fisiológicos del duraznero (P. persica (L.) Batsch) en el trópico alto. Una revisión. Rev. U.D.C.A Act. \& Div. Cient. (Colombia). 17(2):401-411.

24. QUEVEDO, E.G.; ARÉVALO, G.M.E.; CANCINO, E.G.O. 2012. Determination of a mathematical model to estimate the area and dry weight of the leaf limbo of P. persica cv. Jarillo. Rev. Fac. Nal. Agr. (Colombia). 65(2):6605-6613.

25. QUEVEDO, G.E.; DARGHAN A., E.; FISCHER, G. 2017. Clasificación de variables morfológicas del duraznero ( $P$. persica L. Batsch) 'Jarillo' en la montaña santandereana colombiana mediante análisis discriminante lineal. Rev. Col. Cienc. Hortic. 11(1):39-47.

26. RAZQUIN, C.J.; MADDONNI, G.A.; VEGA, C.R.C. 2017. Estimación no destructiva del área foliar en plantas individuales de maíz (Zea mays L.) creciendo en canopeos. AgriScientia (Argentina). 34:27-38.

27. ROBERTI, J.O.; BAS NAHAS, S.S.; ROMERO, J.I.; INTERDONATO, R.; BUDEGUER, R.F.; AMADO, M.E.; RODRIGUEZ R., J.A.; ROMERO, E.R. 2014. Métodos no destructivos de estimación del área foliar de hojas individuales en dos híbridos de sorgo dulce [Sorghum bicolor (L.) Moench]. Nota técnica, Rev. Ind. y Agríc. de Tucumán (Argentina). 91(1):2731.

28. TONDJO, K.; BRANCHERIAU, L.; SABATIER, S.A.; KOKUTSE, A.D.; AKOSSOU, A.; KOKOU, K.; FOURCAUD, T. 2015. Non-destructive measurement of leaf area and dry biomass in Tectona grandis. Trees (Alemania). 29:1625-1631.

29. UNIGARRO-MUÑOZ, C.A.; HERNÁNDEZ-ARREDONDO, J.D.; MONTOYA-RESTREPO, E.C.; MEDINARIVERA, R.D.; IBARRA- RUALES, L.N.; CARMONAGONZÁLEZ, C.Y.; FLÓREZ-RAMOS, C P. 2015. Estimation of leaf area in coffee leaves (Coffea arabica L.) of the Castillo ${ }^{\circledR}$ variety. Bragantina, Campinas (Brasil). 7(4):412-415.

30. VALBUENA, N.; PARRAGA, C.; LINARES, L.; RAMOS, J.; JUNCO, J. 2016. Modelos de estimación de área foliar a partir de observaciones morfológicas en Brachiaria brizantha cv. Toledo. Rev. Unell. Cienc. Tec. (Venezuela). 34: 40-44.

31. VIVEROS-VIVEROS, H.; CAMARILLO-LUNA, A.R.; SÁENZ-ROMERO, C.; ARMANDO APARICIORENTERÍA, A. 2013. Variación altitudinal en caracteres morfológicos de Pinus patula en el estado de Oaxaca (México) y su uso en la zonificación altitudinal. Bosque (Chile), 34(2):173-179.

Recibido: Agosto 4 de 2017

Aceptado: Septiembre 19 de 2017

Cómo citar:

Quevedo García, E.; Cancino Escalante, G.O.; Barragán Torres, A.R. 2017. Modelos de regresión para estimar el peso seco de órganos y área del limbo del duraznero, variedad Jarillo. Rev. U.D.C.A Act. \& Div. Cient.20(2): 299-310. 